\title{
Recent epidemiologic trends in periodontitis in the USA
}

\author{
Paul I. Eke ${ }^{1}$ | Wenche S. Borgnakke ${ }^{2}$ | Robert J. Genco ${ }^{3}$ \\ ${ }^{1}$ Division of Population Health, National Center for Chronic Disease Prevention and Health Promotion, Centers for Disease Control and Prevention (CDC), \\ Atlanta, Georgia, USA \\ ${ }^{2}$ Department of Periodontics and Oral Medicine, University of Michigan School of Dentistry, Ann Arbor, Michigan, USA \\ ${ }^{3}$ (Formerly) Distinguished Professor of Oral Biology and Microbiology; Director, UB Center for Microbiome Research, State University of New York \\ (SUNY), University at Buffalo, Amherst, New York, USA
}

Correspondence

Paul I. Eke, Division of Population Health, National Center for Chronic Disease and Health Promotion, Centers for Disease Control and Prevention (CDC), MS-45 Atlanta, GA 30341, USA.

Email: peke@cdc.gov

\section{1 | INTRODUCTION}

Population-based data are fundamental to understanding the distribution and determinants of disease in populations, and their application to prevention programs. The most important trends in the epidemiology of periodontitis in the last decade have centered around improvements in population survey methodologies and statistical modeling of periodontitis for US adult populations, including the suggestion of a new classification of periodontitis cases for surveillance. Most of these advancements have occurred as the direct result of work by the joint initiative known as the Periodontal Disease Surveillance Project by the Centers for Disease Control and Prevention and the American Academy of Periodontology. Information and findings accruing from these recent advancements have collectively and directly manifested themselves in important results, such as important revisions of our knowledge of the epidemiology of periodontitis in US adults.

Periodontitis is an important public health problem in the USA. This is illustrated by the US Department of Health and Human Services designating oral health as one of 42 health topic areas identified in Healthy People 2020. ${ }^{1}$ Of the 33 objectives within oral health in the future is the goal "OH-5. Reduce the proportion of adults aged 45-74 years with moderate or severe periodontitis" set forth by the US Department of Health and Human Services in the 2010 report called Healthy People $2020 .^{2}$ Notably, the goal was first set in 2010, but its numeric value for reduction of the prevalence of moderate or severe periodontitis in adults aged 45-74 years was later revised and operationalized on the basis of analyses of data from the 2009-2010 National Health and Nutrition Examination Surveys collected in accord with the new full-mouth periodontal examination protocol. Applying the novel Centers for Disease Control and Prevention and the American Academy of Periodontology periodontitis case definitions, the goal is currently a reduction in periodontal diseases from $47.5 \%$ to $40.8 \%{ }^{2}$

Assessing the progress towards this goal requires ongoing national disease surveillance and health promotion activities that include monitoring periodontitis prevalence. Federally funded national surveys, such as the National Health and Nutrition Examination Surveys, have been the only source of nationally representative data on periodontal diseases. Over the years, these surveys have undergone considerable modification to improve the validity of information resulting from them, while still keeping the cost of data collection as low as possible. The earliest of these surveys, namely the 1960-1962 Health Examination Survey and the 1971-1974 National Health and Nutrition Examination Survey, assessed periodontal status by visual inspection only. By contrast, subsequent surveys, namely, the 1985-1986 National Survey of Employed Adults and Seniors instituted by the National Institute of Dental and Craniofacial Research, the 1988-1994 National Health and Nutrition Examination Survey III, and the 1999-2004 National Health and Nutrition Examination Survey, used periodontal probing measurements to assess periodontal probing depth and gingival recession around teeth. These assessments followed various partial-mouth periodontal examination protocols, ${ }^{3-6}$ ranging from collecting measurements from two randomly selected quadrants of the mouth assessing periodontal probing depth and clinical attachment loss at two sites per tooth (mesio-buccal and mid-buccal sites) in the National Health and Nutrition Examination Survey III and the National Health and Nutrition Examination Survey 1999-2000, to assessing three sites (mesio-buccal, mid-buccal, and disto-buccal sites) in the National Health and Nutrition Examination Survey 2001-2004. However, these partial-mouth periodontal examination protocols harbor inherent errors because periodontitis is a site-specific disease and therefore is not evenly distributed in the mouth. 
Thus, prevalence estimates from surveys using partial-mouth periodontal examination protocols underestimate disease in the population; and this underestimation can be significant in National Health and Nutrition Examination Surveys. ${ }^{6}$ Periodontal examinations in National Health and Nutrition Examination Surveys ceased after the 2003-2004 data collection cycle.

Following work by the Centers for Disease Control and Prevention and the American Academy of Periodontology Periodontal Disease Surveillance Project, National Health and Nutrition Examination Survey surveillance of periodontal disease was reinstated in 2009. The 2009-2014 survey protocol was based on evidence from pilot studies that demonstrated the feasibility of using a full-mouth periodontal examination protocol for surveillance of periodontitis in National Health and Nutrition Examination Surveys. Thus, the 2year National Health and Nutrition Examination Survey 2009-2010 survey cycle, later expanded to the National Health and Nutrition Examination Survey 2011-2012 and the National Health and Nutrition Examination Survey 2013-2014 survey cycles, consisted of the first national probability sample surveys to use the full-mouth periodontal examination protocol, collecting periodontal probing measurements from six sites per tooth for all teeth (except third molars) in US adults. Using this gold standard protocol of periodontal measurements from six sites per tooth for all nonthird molar teeth to identify periodontitis cases vastly improved the validity of estimates for periodontitis harvested from US national surveys. The National Health and Nutrition Examination Survey protocols for the 2009-2010, 2011-2012, and 2013-2014 cycles dictated that dentate participants aged 30-79 years would be eligible for the periodontal examination. This age interval was determined to ensure cost-effectiveness of the resource allocation within National Health and Nutrition Examination Surveys. The lower age limit of 30 years was determined to include only participants most likely to suffer from periodontitis, as people younger than 30 years are less likely to have periodontitis. Only a few participants aged 80 years or older were expected to be dentate, so their inclusion would risk producing data which would not represent this older population group nationally. Consequently, when an expression representing the concept of 30 years or older is used in this review, it refers to the age group of 30-79 years.

Any figures and results reported by numbers described in the text that are not designated as derived from any previous publication have been generated specifically for this review based on data from the National Health and Nutrition Examination Surveys 2009-2014.

Data obtained from these three 2-year survey cycles in combination with other codetermined data of sociodemographic, behavioral and co-occurring morbidities provide unprecedented and unique data to re-examine the epidemiologic characteristics of periodontitis in US adult populations and their relationships with several comorbid conditions. The trust in the accuracy of the data is further supported by thorough examiner training and calibration of clinical examiners against the gold standard examiner, and the subsequent quality assurance emanating from these procedures. ${ }^{7}$ Importantly, for the first time in National Health and Nutrition Examination Survey history, sufficient numbers of non-Hispanic Asians were sampled in the 2011-2012 cycle to provide reliable estimates of their periodontitis prevalence, which further heightens the accuracy of the estimates of periodontitis prevalence. ${ }^{8}$

\section{2 | DEVELOPMENT OF STANDARD CASE DEFINITIONS FOR THE SURVEILLANCE OF PERIODONTITIS}

The historical lack of a standard case definition for surveillance of periodontitis that prevented any meaningful comparisons of findings from different research groups was addressed as a complementary, but pivotal, issue to the survey issue. This void has been a major obstacle in determining, comparing, and pooling estimates for the prevalence of periodontitis from different studies in different populations and countries, as well as between the same populations over time, and comparing changes in periodontitis prevalence over time with changes in other populations. In response to the need for a global standard case definition for surveillance of periodontitis, the Centers for Disease Control and Prevention and the American Academy of Periodontology initiative undertook extensive studies and consultations with experts to arrive at case definitions for none/ mild, moderate, and severe periodontitis for use in surveillance. ${ }^{9}$ Further, separate categories for no and mild periodontitis were proposed in $2012 .^{10}$

Applying these stringent periodontitis case definitions ensures that cases identified by the definitions indeed do have disease. To minimize the potentially erroneous effect of gingival recession, which may not be a consequence of disease but rather of vigorous toothbrushing, on the accuracy of the probing depth measurements, both clinical attachment loss and periodontal probing depth are used in this classification. Although clinical attachment loss is considered a more accurate measure for periodontitis than periodontal probing depth, and clinical attachment loss is accepted as the gold standard for disease severity and progression, use of clinical attachment loss alone could mistakenly include some periodontally healthy sites because attachment loss can accompany noninflammatory gingival recession. In addition, these definitions were based on moderate agreement in the literature that clinical attachment loss of $\geq 6 \mathrm{~mm}$ is a reasonable cutoff point to differentiate severe from moderate periodontitis; the latter term is usually applied to a clinical attachment loss of 4-5 mm or less. Moderate periodontitis could mean periodontitis in which pocketing and attachment loss are not yet sufficiently severe to threaten the loss of teeth. Hence, this case definition requires at least two sites with periodontal probing depth $\geq 5 \mathrm{~mm}$ in addition to clinical attachment level, in part to exclude cases that have been treated successfully but still have attachment loss, or have attachment loss not resulting from periodontitis. Moreover, measurements from interproximal sites are used in contrast to mid-buccal and mid-lingual sites because the disease usually begins at and is 
TAB LE 1 Centers for Disease Control and Prevention and the American Academy of Periodontology periodontitis case definitions for use in surveillance and subsequent categories used in reporting by the Centers for Disease Control and Prevention and the American Academy of Periodontology Project

\begin{tabular}{|c|c|c|c|}
\hline \multirow[b]{2}{*}{$\begin{array}{l}\text { Periodontitis case } \\
\text { definitions }\end{array}$} & \multirow{2}{*}{$\begin{array}{l}\text { Criteria }^{10} \\
\text { Severe vs moderate vs mild vs none }\end{array}$} & \multicolumn{2}{|c|}{$\begin{array}{l}\text { Subsequently derived } \\
\text { periodontitis categories }\end{array}$} \\
\hline & & $\begin{array}{l}\text { Total }^{\mathrm{a}} \text { vs } \\
\text { none }\end{array}$ & $\begin{array}{l}\text { Severe vs } \\
\text { nonsevere }\end{array}$ \\
\hline Severe & $\begin{array}{l}\geq 2 \text { interproximal sites with } \geq 6 \mathrm{~mm} \text { clinical at- } \\
\text { tachment loss (not on the same tooth) } \\
\text { AND } \\
\geq 1 \text { or more interproximal site }(\mathrm{s}) \text { with } \geq 5 \mathrm{~mm} \\
\text { periodontal probing depth }\end{array}$ & Severe & Severe \\
\hline Moderate & $\begin{array}{l}\text { Among those who did not meet the severe } \\
\text { periodontitis case definition: } \\
\geq 2 \text { interproximal sites with } \geq 4 \mathrm{~mm} \text { clinical at- } \\
\text { tachment loss (not on the same tooth) } \\
\text { OR } \\
\geq 2 \text { interproximal sites with periodontal prob- } \\
\text { ing depth } \geq 5 \mathrm{~mm} \text { (not on the same tooth) }\end{array}$ & Moderate & Moderate \\
\hline Mild & $\begin{array}{l}\text { Among those who met neither the severe nor } \\
\text { moderate periodontitis case definitions: } \\
\geq 2 \text { interproximal sites with } \geq 3 \mathrm{~mm} \text { clinical at- } \\
\text { tachment loss } \\
\text { AND } \\
\text { ( } \geq 2 \text { interproximal sites with } \geq 4 \mathrm{~mm} \text { periodontal } \\
\text { probing depth (not on the same tooth) } \\
\text { OR } \\
1 \text { site with } \geq 5 \mathrm{~mm} \text { periodontal probing depth) }\end{array}$ & Mild & Mild \\
\hline None & $\begin{array}{l}\text { Meets neither the severe nor moderate nor } \\
\text { mild periodontitis case definitions }\end{array}$ & None & None \\
\hline
\end{tabular}

${ }^{\text {aT }}$ Total periodontitis was defined as severe or moderate periodontitis in the 2010 report on accuracy of estimates based on earlier National Health and Nutrition Examination Survey protocols $^{6}$ because the Centers for Disease Control and Prevention and the American Academy of Periodontology periodontitis case definitions consisted of only the categories of severe, moderate, and mild/none at the time. ${ }^{9}$ The mild category was separated out from the original mild/none periodontitis category in 2012. ${ }^{10}$ Total periodontitis*(= "any" periodontitis): severe or moderate or mild periodontitis. ${ }^{8,11-17}$ Nonsevere periodontitis (= "other" periodontitis): moderate or mild periodontitis. ${ }^{17}$ most severe at interproximal sites. Importantly, these case definitions are intended for use in field surveys and not for clinical practice. The Centers for Disease Control and Prevention and the American Academy of Periodontology case definitions are operationalized in Table 1.

A recent study has validated these case definitions relative to clinical observations and report strong correlations between the periodontal inflamed surface area and case status based on this classification. $^{18}$

\section{GINGIVITIS}

Gingivitis is nearly ubiquitous with up to $90 \%$ in any affected population worldwide. ${ }^{19}$ The 2017 World Workshop proposed case definitions for gingival health and for gingivitis for use in surveillance. ${ }^{20}$ Nonetheless, because no assessments of gingival health, such as bleeding on probing, coloring, or swelling, were included in the protocol for 2009-2014 National Health and Nutrition Examination Surveys to examine for gingivitis, no further description will be provided regarding the prevalence of this reversible disease that only progresses to periodontitis in especially susceptible individuals. ${ }^{21,22}$

\section{Application of the Centers for Disease Control and Prevention and the American Academy of Periodontology periodontitis case definitions as global standards}

The Centers for Disease Control and Prevention and the American Academy of Periodontology case definitions are gradually being adopted globally as the standard for reporting the prevalence of periodontitis, and have been used in a multitude of studies around the world. Of great importance is that the Centers for Disease Control and Prevention and the American Academy of Periodontology periodontitis case definitions are among those that should be applied when reporting the prevalence of periodontitis, as per a consensus report by experts in Europe and the USA outlining standard reporting of periodontitis prevalence in the future in their document "Proposed standards from the Joint EU/USA Periodontal Epidemiology Working Group". ${ }^{23}$ 


\section{3 | REVISED ESTIMATES OF PERIODONTITIS PREVALENCE IN US ADULTS}

Applying the Centers for Disease Control and Prevention and the American Academy of Periodontology case definition for periodontitis to the pooled data obtained during the 6 years that National Health and Nutrition Examination Surveys followed the protocol prescribing periodontal probing at six sites around all nonthird molar teeth, namely the period 2009-2014, resulted in significant revisions of the estimates of prevalence of periodontitis in US adults. Complete clinical periodontal examinations, sociodemographic, health behavior, and comorbidity data were available for 10,683 participants, constituting a nationally representative sample representing a weighted population of $\sim 143.8$ million civilian noninstitutionalized dentate US adults aged 30 years or older.

This ensures that analyses of these data will result in the most accurate estimates of the prevalence of periodontitis and its associated risk factors currently in existence in the world. Hence, findings from our analyses of these data function as a benchmark for surveys conducted in the rest of the world. Our reporting of the prevalence of periodontitis using both the Centers for Disease Control and Prevention and the American Academy of Periodontology case definitions, as well as various thresholds of periodontal probing depth and clinical attachment loss and units of population, teeth, and sites, further enhances the utility of the results, in addition to making the data publicly available.

\section{1 | Total (any) periodontitis}

Notably, the prevalence of periodontitis in dentate US adults aged 30-79 years was significantly higher than previously reported. Overall, $42.2 \%$ (standard error \pm 1.4 ) of US dentate adults aged $\geq 30$ years had some category of periodontitis, consisting of $7.8 \%$ with severe periodontitis and $34.4 \%$ with nonsevere periodontitis (ie, moderate and mild periodontitis combined) (Figure 1).

Furthermore, the prevalence of periodontitis by severity and by age group, and by smoking status, respectively, is shown in Figures 2 and 3.

The prevalence of periodontitis among the oldest age group, 65-79 years, is described in more detail in a separate report based on analyses of data from the 2009-2010 and 2011-2012 National Health and Nutrition Examination Survey cycles. ${ }^{16}$

\section{2 | Periodontal probing depth}

At the probing site level, the mean periodontal probing depth was $1.5 \mathrm{~mm}$. About $37.5 \%$ of adults had $>1$ site with a periodontal probing depth $>4 \mathrm{~mm}$, affecting on average $3.3 \%$ of sites and $9.1 \%$ of teeth per person. ${ }^{17}$ Overall, the mean proportion of sites with periodontal probing depth $>4 \mathrm{~mm}$ was $3.3 \%$. At the tooth level, $29.3 \%$ had a periodontal probing depth of $>4 \mathrm{~mm}$ in $>5 \%$ of their teeth, whereas $10.5 \%$ had $>30 \%$ of their teeth affected by a periodontal

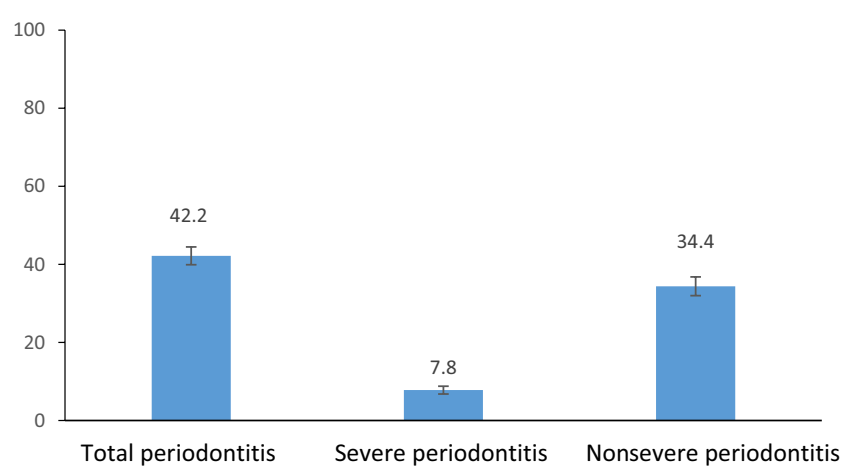

FIGURE 1 Prevalence of total, severe, and nonsevere periodontitis in dentate adults aged $30-79$ years: National Health and Nutrition Examination Survey, 2009-2014 ( $N=10,683$ ). Total (any) periodontitis: severe, moderate, or mild periodontitis; nonsevere periodontitis: moderate or mild periodontitis

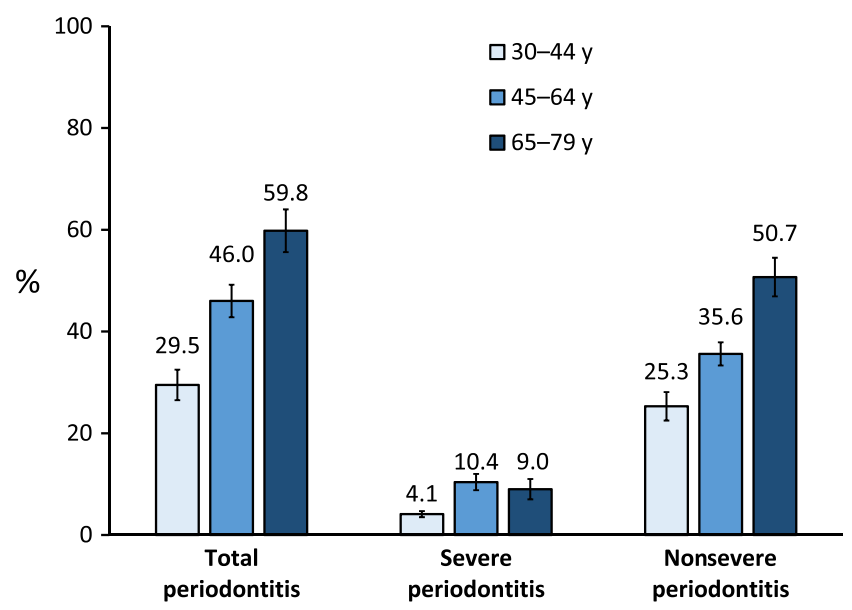

FIGURE 2 Prevalence of total, severe, and nonsevere periodontitis by age group in dentate adults aged $30-79$ years: National Health and Nutrition Examination Survey, 2009-2014 ( $N=10,683$ ). Total (any) periodontitis: severe, moderate, or mild periodontitis; nonsevere periodontitis: moderate or mild periodontitis

probing depth $>4 \mathrm{~mm}$. The overall mean proportion of teeth with a periodontal probing depth of $>4 \mathrm{~mm}$ was $9.1 \%$. However, almost half of older adults (ie, those aged $\geq 65$ years) had at least one site with a periodontal probing depth of $\geq 4 \mathrm{~mm}$. An estimated $15.0 \%$ of adults had a periodontal probing depth of $>4 \mathrm{~mm}$ at $>5 \%$ of all sites and $2.7 \%$ at $>30 \%$ of all sites. ${ }^{17}$

\subsection{Clinical attachment loss}

The overall US population mean clinical attachment loss was $1.7 \mathrm{~mm} .{ }^{17}$ About $89 \%$ had $>1$ site with clinical attachment loss of $>3 \mathrm{~mm}$ with an average of $19.0 \%$ of sites per person and an average of $37.1 \%$ of teeth per person affected.

Overall, an estimated $58.3 \%$ had $>3 \mathrm{~mm}$ clinical attachment loss in $>5 \%$ of sites; and the mean proportion of sites with $>3 \mathrm{~mm}$ clinical attachment loss was $19.0 \%$. At the tooth level, $80.8 \%$ of adults had a clinical attachment loss of $>3 \mathrm{~mm}$ in $>5 \%$ of their teeth, while $47.3 \%$ had 


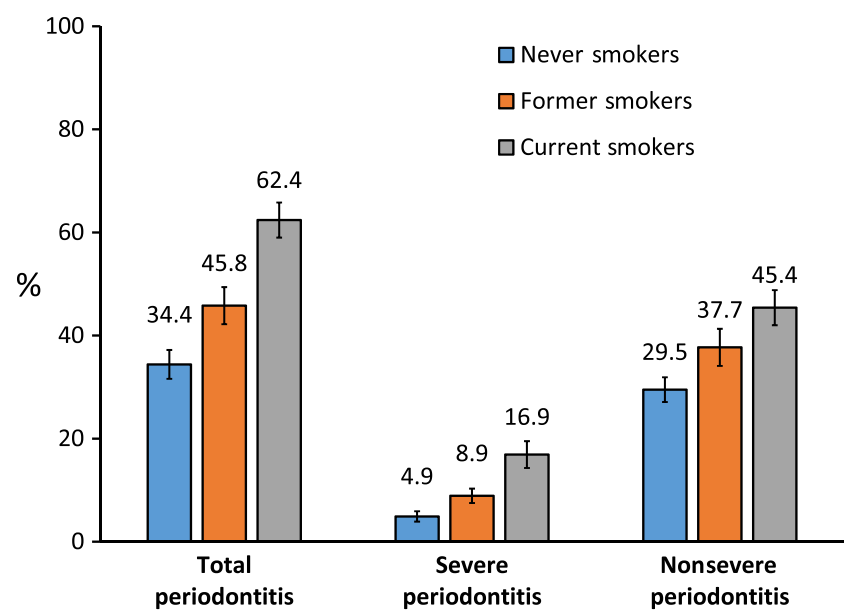

FIGURE 3 Prevalence of total, severe, and nonsevere periodontitis by smoking status in dentate adults aged 30-79 years: National Health and Nutrition Examination Survey, 2009-2014 ( $N=10,683$ ). Total (any) periodontitis: severe, moderate, or mild periodontitis; nonsevere periodontitis: moderate or mild periodontitis

$>30 \%$ of their teeth affected by a clinical attachment loss of $>3 \mathrm{~mm}$. The mean proportion of teeth with $>3 \mathrm{~mm}$ clinical attachment loss was $37.1 \%$. Among older adults (ie, those aged $\geq 65$ years), almost two-thirds (62.3\%) had at least one site with $\mathrm{a} \geq 5 \mathrm{~mm}$ clinical attachment level. ${ }^{17}$

\section{4 | TRENDS IN PERIODONTITIS PREVALENCE IN US ADULTS}

Because the former nationally representative surveys all used partial-mouth periodontal examinations and thereby potentially missed a significant proportion of the disease, possibly misclassifying more than half of participants, ${ }^{6}$ any difference in the prevalence calculated is more likely to be a consequence of applying different examination protocols than reflecting any actual change in the prevalence of periodontitis. Hence, it is not possible to know whether periodontitis prevalence is increasing or decreasing in the USA. However, because people live longer and keep their teeth for longer as they grow older, and because periodontitis is defined as a chronic disease that accumulates in an individual during their lifetime, it is reasonable to speculate that there are increasing numbers of people and teeth with periodontitis.

\section{5 | RISK INDICATORS FOR PERIODONTITIS}

Similarly, using data from the 2009-2014 National Health and Nutrition Examination Surveys, we reassessed risk indicators for periodontitis after controlling for sociodemographic, behavioral, and comorbid conditions using multivariable logistic regression modeling. Figure 4 illustrates the risk ratios for having severe periodontitis.

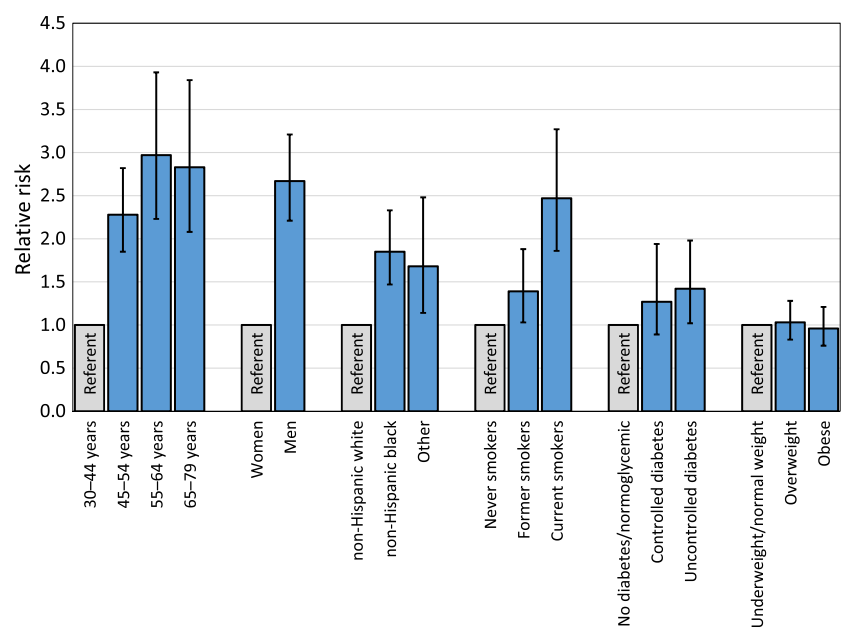

FIGURE 4 Relative risk for severe periodontitis in dentate adults aged 30-79 years by age group, sex, race/ethnicity, cigarette smoking habits, diabetes status, and obesity status: National Health and Nutrition Examination Survey, 2009-2014 ( $N=10,683)$. The following groups were used for comparison, that is, they were assigned the relative risk value of 1: age 30-44 years (age group), female (sex), non-Hispanic white (race/ethnicity), never smoker (cigarette smoking habits), no diabetes/normoglycemic (diabetes status), and normal weight

Consistent with what was hitherto known, the likelihood of having total (any) or nonsevere periodontitis increased steadily with increasing age. However, this was not the case for the prevalence of severe periodontitis, which did not increase with age (Figure 4). As an aside, since the prevalence of mild periodontitis also did not consistently increase with age (not shown), ${ }^{17}$ the increase in periodontitis prevalence by age is primarily driven by the moderate disease. ${ }^{17}$ The likelihood of having periodontitis was two times greater among males compared with females, with the highest probability observed for severe periodontitis (adjusted prevalence ratio $\mathrm{aPR}=2.68 ; 2.22-3.23$ ) (Figure 4 ). Periodontitis was most likely present among Hispanics ( $\mathrm{aPR}=1.38 ; 1.26-1.52)$ and nonHispanic blacks (aPR $=1.35 ; 1.22-1.50)$; and severe periodontitis was most likely among non-Hispanic blacks ( $a P R=1.82 ; 1.44-2.31)$ compared with non-Hispanic whites (Figure 4). Adults who have less than a high school education were more likely to have periodontitis, with the greatest probability observed for severe periodontitis ( $a P R=1.63,1.26-2.12$ ) (Figure 4); but the probability was also sizeable for nonsevere periodontitis ( $\mathrm{PPR}=1.29,1.15-1.45)$ compared with those with more than a high school education. The highest probability for severe periodontitis was observed among adults with income levels at $100 \%-199 \%$ of the federal poverty level (aPR $=1.82,1.22-2.71)$ (Figure 4), while the highest probability for nonsevere periodontitis was observed among the poorest at $<100 \%$ of the federal poverty level $(a P R=1.44,1.26-1.56)$. Overall, there was a steady increase in the likelihood of periodontitis with increasing poverty (ie, decreasing federal poverty level) (not shown). Periodontitis was significantly more likely among current and former smokers compared with nonsmokers. The 
likelihood for periodontitis was highest among current smokers $(a P R=1.54,1.45-1.65)$, and smoking was strongly associated with the severe form of periodontitis ( $\mathrm{aPR}=2.46,1.87-3.24)$ (Figure 4). Among people with diabetes, periodontitis was more likely only in those with uncontrolled diabetes, specifically pertaining to severe periodontitis (aPR = 1.42, 1.02-1.98) (Figure 4). Periodontitis was not significantly associated with obesity status. Of note, severe periodontitis was neither associated with overweight nor obesity (Figure 4). Finally, the likelihood of severe periodontitis was greatest in adults aged $<65$ years, in males, in non-Hispanic blacks, and in current cigarette smokers when compared with non-severe periodontitis.

When stratified by sex, severe periodontitis was more likely among females aged 65 years and older. Periodontitis was equally likely in female former smokers and nonsmokers. In females, periodontitis and non-severe periodontitis were not significantly associated with diabetes status. By contrast, all levels of severity of periodontitis were more likely among males with uncontrolled diabetes when compared with males without diabetes. Finally, when compared with people with non-severe periodontitis, the likelihood of severe periodontitis was significantly greater in males of all age groups and education status, and for non-Hispanic blacks and current smokers, regardless of sex.

Further analyses of periodontitis and diabetes also suggest that the duration of diabetes did not significantly correlate with the likelihood of periodontitis after adjusting for all covariates (Wald $F$ test, $P>0.05$ ). Similarly, no trend was observed between periodontitis and fasting glucose level among people with diabetes (Wald $F$ test, $P>0.05$ ). In females without diabetes, total periodontitis and non-severe periodontitis (but not severe periodontitis) significantly increased with increasing fasting glucose level $(P<0.05)$. In males without diabetes, no trend was observed for any level of severity of periodontitis and increasing fasting glucose level $(P>0.05)$. Periodontitis was significantly associated with increasing levels of glycated hemoglobin in both males and females without diabetes $(P<0.05)$. Among people with diabetes, only severe periodontitis was significantly correlated with increasing levels of glycated hemoglobin in males.

\section{6 | ESTIMATING PERIODONTITIS AT STATE AND LOCAL LEVELS}

Until recently, population estimates of periodontitis were virtually nonexistent at state and local levels, even though most public health interventions are administrated at state and local populations. This is attributable to the lack of resources within existing state-based oral health surveillance systems to support clinically assessed periodontal measurements. Thus, for now, population-based surveillance at state and local levels will require the development of less resource-demanding measures that can be integrated into existing surveillance systems. Alternatively, multilevel statistical modeling can generate estimates of the burden of periodontitis at state and local levels.

\section{1 | Direct estimation of periodontitis at state and local levels}

Currently, there are several ongoing interview-based surveys at national, state, and local levels that can be explored for surveillance of periodontitis. In the USA, measures derived from responses to self-report questions incorporated into state interview surveys (eg, the state-based Behavioral Risk Factor Surveillance System) have been successful in generating actionable public health data for several chronic diseases and risk factors at state and local levels. ${ }^{24,25}$ Thus, since 2006, the Centers for Disease Control and Prevention and the American Academy of Periodontology Periodontal Disease Surveillance Project has been investigating the potential use of self-report measures for estimating the prevalence of periodontitis in adult populations. Among ways to identify appropriate, relevant, and validated questionnaire items for potential use for self-report was the conduction of a systematic review of studies that sought to validate self-reported measures for periodontal disease. ${ }^{26}$ Another avenue was to examine items used in prior work by members of the Centers for Disease Control and Prevention and the American Academy of Periodontology initiative workgroup who had conducted epidemiologic studies encompassing both self-reported and clinically assessed periodontitis, hence allowing assessment of the validity of the items as proxies for actual clinically assessed periodontal disease. ${ }^{27-29}$

The Centers for Disease Control and Prevention and the American Academy of Periodontology initiative identified eight selfreport measures that are promising for use in estimating the prevalence of periodontitis in adult populations as their responses are correlated with clinical measures of periodontitis. ${ }^{11}$ The questions in English are displayed in Table 2, whereas the Spanish version may be found in the 2009 report. ${ }^{11}$

Using multivariable prediction models, the performance of these self-report measures was evaluated in the Australian National Adult Oral Health Survey ${ }^{30}$ and in a convenience sample of adults in Brazil. ${ }^{31}$ Importantly, the items were cognitively tested, and a pilot field was tested in one National Health and Nutrition Examination Survey stand before being incorporated in the full National Health and Nutrition Examination Survey. ${ }^{11}$

In these preliminary assessments, response rates to all self-report questions in at-home interviews were high, namely $>95 \%$. All self-reported measures were independently associated with periodontitis, except for the use of mouthwash. Self-reported questions had no significant correlations with one another, with the exception of the use of mouthwash and evidence of bone loss. ${ }^{11}$ In multivariable modeling, the combined effects of demographic measures and measures from five self-report questions in detecting unweighted total periodontitis performed at a sensitivity of $84 \%$, specificity of $60 \%$, and receiver operating characteristic of 0.81 . Three questions 
TABLE 2 Questions for self-report of periodontitis identified as promising for potential use in survey-based surveillance in lieu of resource-intensive clinical periodontal examinations ${ }^{11}$

\begin{tabular}{|c|c|c|}
\hline Item number & Item verbatim & Response options \\
\hline 1. & Do you think you might have gum disease? & Yes/no/refused/don't know \\
\hline 2. & $\begin{array}{l}\text { Overall, how would you rate the health of } \\
\text { your teeth and gums? }\end{array}$ & $\begin{array}{l}\text { Excellent/very good/good/ } \\
\text { fair/poor/refused/don't know }\end{array}$ \\
\hline 3. & $\begin{array}{l}\text { Have you ever had treatment for gum } \\
\text { disease such as scaling and root planing, } \\
\text { sometimes called "deep cleaning"? }\end{array}$ & (yes/no/refused/don't know \\
\hline 4. & $\begin{array}{l}\text { Have you ever had any teeth become loose } \\
\text { on their own, without an injury? }\end{array}$ & Yes/no/refused/don't know \\
\hline 5. & $\begin{array}{l}\text { Have you ever been told by a dental } \\
\text { professional that you lost bone around } \\
\text { your teeth? }\end{array}$ & Yes/no/refused/don't know \\
\hline 6. & $\begin{array}{l}\text { During the past } 3 \text { months, have you no- } \\
\text { ticed a tooth that doesn't look right? }\end{array}$ & Yes/no/refused/don't know \\
\hline 7. & $\begin{array}{l}\text { Aside from brushing your teeth with a } \\
\text { toothbrush, in the last } 7 \text { days, how many } \\
\text { times did you use mouthwash or other } \\
\text { dental rinse product that you use to treat } \\
\text { dental disease or dental problems? }\end{array}$ & $\begin{array}{l}\text { Number of days: } \\
\text { refused }\end{array}$ \\
\hline 8. & $\begin{array}{l}\text { Aside from brushing your teeth with } \\
\text { a toothbrush, in the last } 7 \text { days, how } \\
\text { many times did you use dental floss or } \\
\text { any other device to clean between your } \\
\text { teeth? }\end{array}$ & $\begin{array}{l}\text { Number of days: } \\
\text { refused }\end{array}$ \\
\hline
\end{tabular}

performed at a sensitivity of $95 \%$, specificity of $28 \%$, and receiver operating characteristic of 0.82 in predicting clinical attachment loss of $\geq 3 \mathrm{~mm}$. In validation tests, the two models performed at prediction accuracies of $70 \%$ and $89 \%$, respectively.

These results confirm that self-report measures can perform well in predicting prevalence for periodontitis in the US adult population. Notably, our preliminary findings suggest the performance of these questions may exceed the accuracy of estimates from partial-mouth examination protocols commonly used in surveillance of periodontitis, especially for severe periodontitis. ${ }^{6}$

In 2009, the Centers for Disease Control and Prevention and the American Academy of Periodontology initiative began inclusion of these self-report questions in the at-home interview questionnaire for the National Health and Nutrition Examination Survey. The data from the 2009-2014 cycles will be used to test and validate the performance of these self-report questions against clinically determined periodontitis. The full-mouth examination protocol used in National Health and Nutrition Examination Surveys provides clinical data that will minimize misclassification of periodontitis cases for this validation study. Analyses of the data are underway to generate the model coefficients for use in estimating periodontitis in populations based on responses to self-report questions. The self-report questions are still incorporated in National Health and Nutrition Examination Surveys and will be used for future monitoring of the prevalence of periodontitis and for assessing trends over time. For the first time this will allow trends to be calculated with the assurance of agreement with clinically assessed periodontitis during 2009-2014 once validated.

\section{2 | Model estimation of periodontitis prevalence at state and local levels}

With the availability of comprehensive National Health and Nutrition Examination Survey data and the concurrent availability of local and state data sets, such as those from the US Census and the Behavioral Risk Factor Surveillance system, it is now possible to estimate periodontitis prevalence at state and local levels. In a recent study, multilevel regression and poststratification analyses were used to estimate the prevalence of periodontitis among adults aged 30-79 years at state, county, congressional district, and census tract levels. ${ }^{15}$ This modeling approach used age, race, sex, smoking, and poverty variables to estimate the prevalence of periodontitis as defined by the Centers for Disease Control and Prevention and the American Academy of Periodontology case definitions at the census tract levels, which subsequently were aggregated to larger administrative and geographic areas of interest. This report was the first time any estimation of the prevalence of periodontitis at state and local levels based on nationally representative data was performed in the USA and represents an important adjunct to public health surveillance efforts to identify areas with a high burden of periodontitis. $^{15}$

Based on National Health and Nutrition Examination Survey 2009-2012 data, the model-estimated mean prevalence of periodontitis among states was $45.1 \%$ (median $44.9 \%$ ), and ranged from $37.7 \%$ in Utah to $52.8 \%$ in New Mexico, representing an estimated 15 percentage points disparity in prevalence among states. County estimates ranged from $33.7 \%$ to $68.0 \%$ (mean $46.6 \%$, median $45.9 \%$ ), 
TAB LE 3 Model-based estimates of total and severe periodontitis prevalence by state or district: National Health and Nutrition Examination Survey, 2009-2012 ${ }^{15}$

\begin{tabular}{|c|c|c|}
\hline State or district & $\begin{array}{l}\text { Total \% ( } 95 \% \text { confi- } \\
\text { dence interval) }\end{array}$ & $\begin{array}{l}\text { Severe } \% \text { ( } 95 \% \text { con- } \\
\text { fidence interval) }\end{array}$ \\
\hline Alabama & $47.39(47.26-47.52)$ & $9.80(9.75-9.85)$ \\
\hline Alaska & $43.60(43.32-43.89)$ & $8.72(8.60-8.83)$ \\
\hline Arizona & $47.73(47.62-47.84)$ & $9.46(9.41-9.50)$ \\
\hline Arkansas & $47.23(47.05-47.41)$ & $9.57(9.50-9.63)$ \\
\hline California & $47.80(47.75-47.84)$ & $9.42(9.40-9.43)$ \\
\hline Colorado & $43.29(43.17-43.40)$ & $8.26(8.22-8.30)$ \\
\hline Connecticut & $43.35(43.22-43.47)$ & $8.18(8.14-8.23)$ \\
\hline Delaware & $45.86(45.60-46.11)$ & $9.03(8.93-9.14)$ \\
\hline $\begin{array}{l}\text { District of } \\
\text { Columbia }\end{array}$ & $50.08(49.79-50.37)$ & $11.18(11.04-11.33)$ \\
\hline Florida & $49.47(49.41-49.55)$ & $10.00(9.97-10.03)$ \\
\hline Georgia & $46.41(46.31-46.51)$ & 9.51 (9.47-9.54) \\
\hline Hawaii & $51.10(50.88-51.32)$ & $10.56(10.45-10.66)$ \\
\hline Idaho & $42.72(42.44-43.01)$ & 7.87 (7.77-7.97) \\
\hline Illinois & $44.87(44.80-44.94)$ & $8.79(8.76-8.81)$ \\
\hline Indiana & $44.18(44.07-44.30)$ & $8.58(8.54-8.63)$ \\
\hline lowa & $42.10(41.95-42.25)$ & $7.66(7.61-7.71)$ \\
\hline Kansas & $43.28(43.13-43.42)$ & $8.17(8.11-8.22)$ \\
\hline Kentucky & $45.17(45.03-45.32)$ & $8.89(8.84-8.94)$ \\
\hline Louisiana & $48.21(48.08-48.33)$ & $10.26(10.2-10.31)$ \\
\hline Maine & $42.90(42.63-43.16)$ & 7.90 (7.80-7.99) \\
\hline Maryland & $45.25(45.15-45.35)$ & $8.97(8.93-9.01)$ \\
\hline Massachusetts & $42.80(42.70-42.89)$ & $8.01(7.98-8.05)$ \\
\hline Michigan & $45.13(45.05-45.21)$ & $8.95(8.91-8.98)$ \\
\hline Minnesota & $41.66(41.55-41.77)$ & 7.68 (7.64-7.72) \\
\hline Mississippi & $49.22(49.04-49.42)$ & $10.58(10.5-10.65)$ \\
\hline Missouri & $45.12(45.00-45.24)$ & $8.88(8.83-8.93)$ \\
\hline Montana & $44.29(43.98-44.57)$ & $8.41(8.30-8.52)$ \\
\hline Nebraska & $42.85(42.65-43.02)$ & $8.00(7.93-8.07)$ \\
\hline Nevada & $47.84(47.69-47.98)$ & $9.68(9.63-9.74)$ \\
\hline New Hampshire & $40.51(40.28-40.73)$ & $7.27(7.18-7.36)$ \\
\hline New Jersey & $45.26(45.18-45.33)$ & $8.82(8.79-8.86)$ \\
\hline New Mexico & $52.79(52.60-52.97)$ & $11.32(11.23-11.41)$ \\
\hline New York & $46.59(46.54-46.65)$ & $9.28(9.26-9.30)$ \\
\hline North Carolina & $46.53(46.44-46.62)$ & $9.47(9.43-9.50)$ \\
\hline North Dakota & $42.42(42.11-42.76)$ & 7.85 (7.73-7.98) \\
\hline Ohio & $44.45(44.37-44.54)$ & $8.70(8.66-8.73)$ \\
\hline Oklahoma & $46.98(46.85-47.12)$ & 9.49 (9.44-9.54) \\
\hline Oregon & $43.63(43.48-43.78)$ & $8.12(8.07-8.18)$ \\
\hline Pennsylvania & $44.41(44.34-44.49)$ & $8.56(8.54-8.59)$ \\
\hline Rhode Island & $43.63(43.38-43.88)$ & $8.26(8.17-8.35)$ \\
\hline South Carolina & $47.78(47.67-47.90)$ & $9.91(9.86-9.96)$ \\
\hline South Dakota & $43.55(43.26-43.87)$ & $8.25(8.15-8.37)$ \\
\hline
\end{tabular}

(Continues)
TABLE 3 (Continued)

\begin{tabular}{|llc|}
\hline State or district & $\begin{array}{l}\text { Total \% (95\% confi- } \\
\text { dence interval) }\end{array}$ & $\begin{array}{l}\text { Severe \% (95\% con- } \\
\text { fidence interval) }\end{array}$ \\
\hline Tennessee & $46.18(46.07-46.30)$ & $9.30(9.25-9.35)$ \\
\hline Texas & $48.25(48.19-48.31)$ & $9.86(9.84-9.89)$ \\
\hline Utah & $37.69(37.51-37.86)$ & $6.36(6.31-6.41)$ \\
\hline Vermont & $41.43(41.09-41.77)$ & $7.47(7.35-7.59)$ \\
\hline Virginia & $44.29(44.21-44.36)$ & $8.65(8.62-8.69)$ \\
\hline Washington & $42.92(42.81-43.02)$ & $8.01(7.98-8.05)$ \\
\hline West Virginia & $45.69(45.46-45.92)$ & $8.88(8.79-8.97)$ \\
\hline Wisconsin & $42.71(42.59-42.83)$ & $8.02(7.98-8.06)$ \\
\hline Wyoming & $42.85(42.51-43.24)$ & $8.01(7.89-8.15)$ \\
\hline
\end{tabular}

representing a much larger disparity of 34 percentage points in periodontitis prevalence among counties, a 2-fold difference (not shown). ${ }^{15}$ The mean prevalence of severe periodontitis among states was $8.9 \%$ (median $8.8 \%$ ) and ranged from $6.4 \%$ in Utah to $11.3 \%$ in New Mexico. Among counties, the mean prevalence was $9.2 \%$ and the median $8.8 \%$, ranging from $5.2 \%$ to $17.9 \%$. In other words, in the county with the greatest prevalence of severe periodontitis, that prevalence is more than three times greater than the prevalence in the county with the lowest prevalence (not shown) (Table 3). ${ }^{15}$

The geographic distribution of estimated periodontitis prevalence at state, county, congressional district, and census track levels is presented in Figure $5 .^{15}$

Overall, the greatest burden of periodontitis was observed among southeastern and southwestern states, concentrated in pockets stretching along the southeast, in the Mississippi Delta, along the USA-Mexico border, and among native American reservations. Other areas severely affected by periodontitis were southern Florida, Hawaii, and remote areas of western Alaska. Overall, similar geographic patterns were determined for severe periodontitis. The prevalence of severe periodontitis in each state is shown in Figure 6.

Among older adults (aged $\geq 65$ years), by state we estimated the lowest prevalence of periodontitis in Utah (62.3\%) and New Hampshire (62.6\%), and the highest in New Mexico, Hawaii, and the District of Columbia, each with a prevalence of $>70 \%$. Overall, periodontitis is highly prevalent in this older subpopulation with almost a minimum of two-thirds of dentate older adults affected at any geographic level.

\section{7 | THE DISTRIBUTION OF SEVERE PERIODONTITIS AND PERIODONTISTS IN THE USA}

Multivariable geospatial analysis was used to examine the distribution of periodontists and adults, periodontists vis-à-vis estimated density of adults with severe periodontitis, and their ratios to adults with severe periodontitis, ${ }^{32}$ taking advantage of the locations identified through the National Provider Identifier Registry. ${ }^{33}$ Overall, $~ 60 \%$ of adults aged $>30$ years live within 5 miles of a periodontist, $73 \%$ within 
A

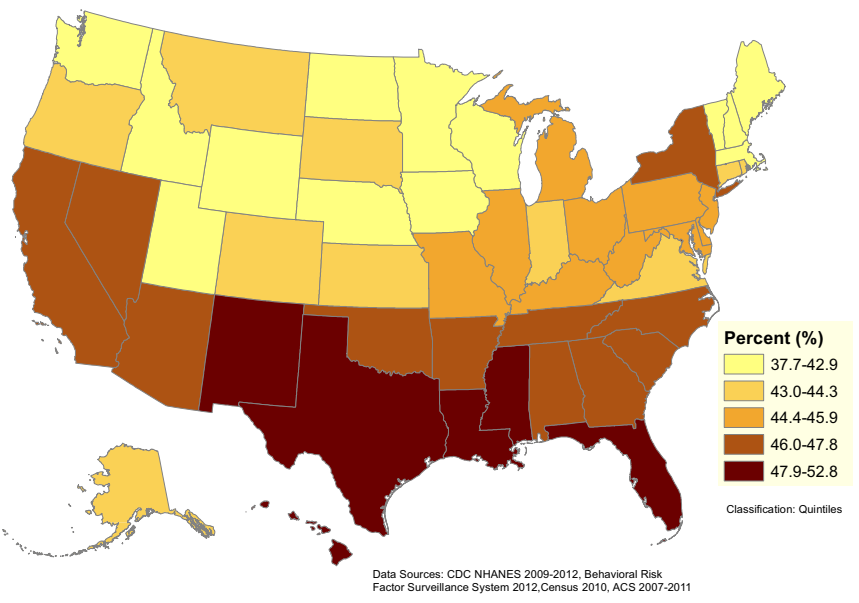

C

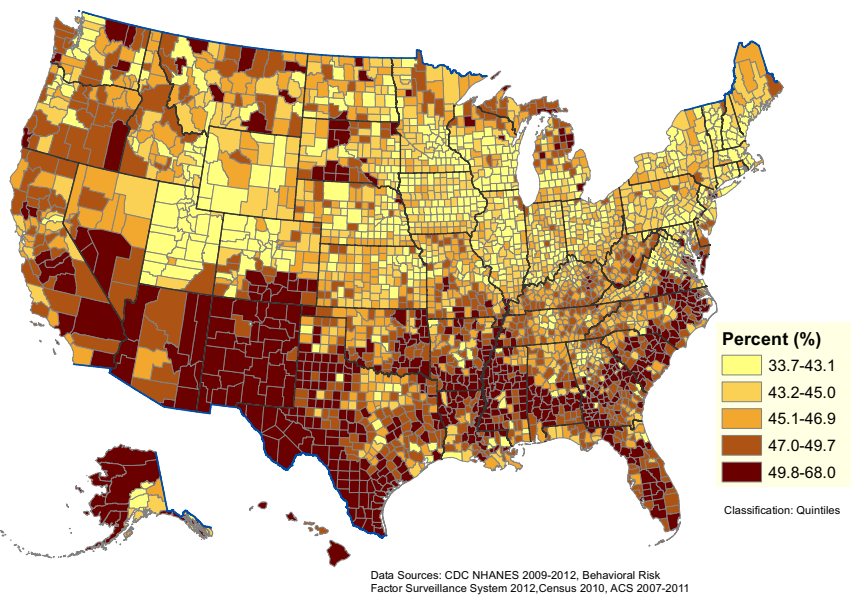

B

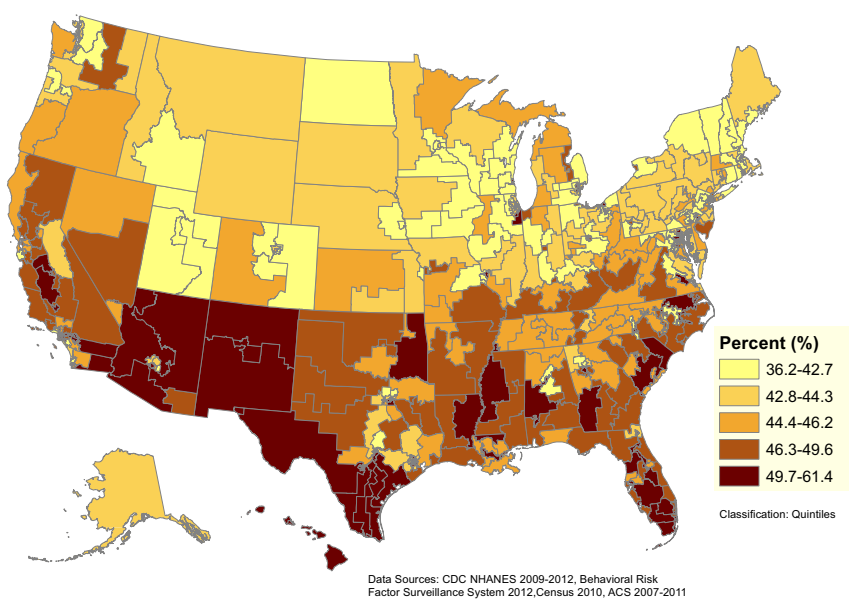

D

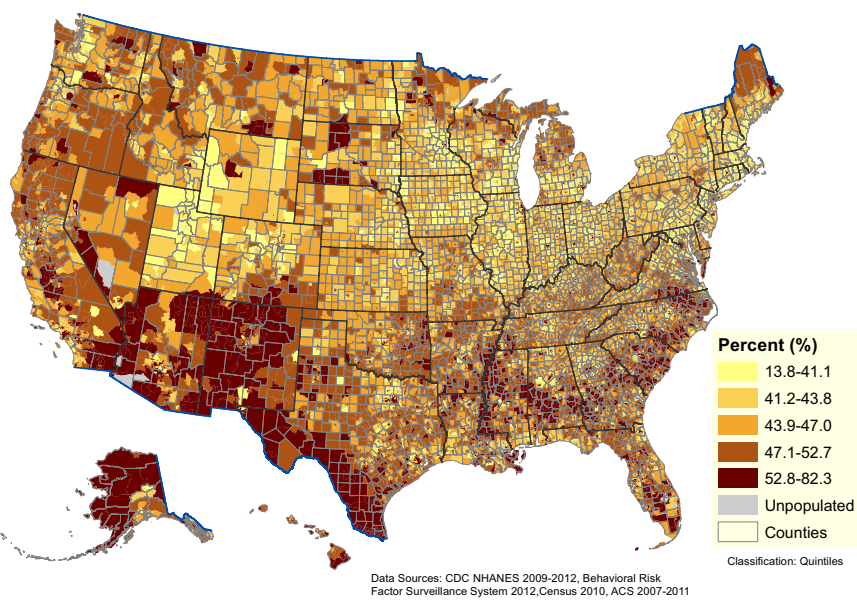

FIGURE 5 Estimates of prevalence of total periodontitis among dentate adults aged 30-79 years by (A) state, (B) congressional district, (C) county, and (D) census tracts: National Health and Nutrition Examination Survey, 2009-2012. Total (any) periodontitis: severe, moderate, or mild periodontitis. ${ }^{15}$ Data sources: Centers for Disease Control and Prevention National Health and Nutrition Examination Survey (National Health and Nutrition Examination Survey) 2009-2012, US Census 2010, and Behavioral Risk Factor Surveillance System 2012

FIGURE 6 Estimates of prevalence of severe periodontitis among dentate adults aged 30-79 years in US states: National Health and Nutrition Examination Survey, 2009-2012. ${ }^{15}$ Data sources: Centers for Disease Control and Prevention National Health and Nutrition Examination Survey (National Health and Nutrition Examination Survey) 2009-2012, US Census 2010, Behavioral Risk Factor Surveillance System 2012, and American Community Survey 2007-2011

\section{Periodontitis Prevalence for Adults Aged 30-79 Years, by States, United States: 2009-2012}

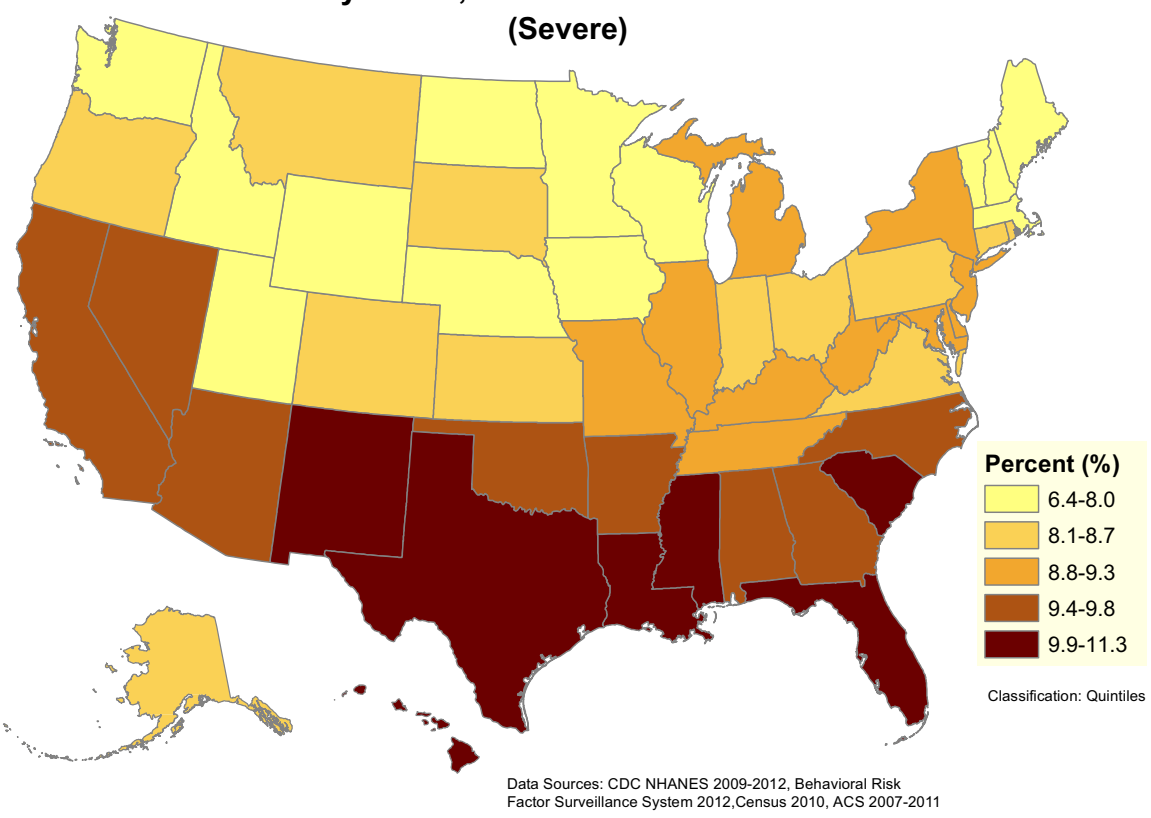


10 miles, 84\% within 20 miles, and 97\% within 50 miles. Proximity to a periodontist varied widely. In urban areas, $95 \%$ of adults resided within 10 miles of a periodontist and $99 \%$ within 20 miles. Only $25 \%$ of adults in rural areas lived within 10 miles of a periodontist. Most periodontists (96.1\%) practiced in urban areas, clustering along the eastern and western coasts and in the Midwest, $3.1 \%$ in urban clusters elsewhere, and $0.8 \%$ in rural areas. Ratios of $<8000$ adults with periodontitis to $>1$ periodontist within 10 miles were mostly clustered in the northeast, central east coast, Florida, west coast, Arizona, and Midwest.

\section{CONCLUSIONS}

Overall, significant progress has been made in laying the groundwork for future data for epidemiologic studies of periodontitis. Importantly, a much improved surveillance framework and data standards have been established that have resulted in revision of the burden of periodontitis in US adults. Replication of these standard surveillance protocols over time will allow reporting of trends in periodontitis prevalence over time. Efforts to determine true trends in periodontitis have been hampered by the different and inconsistent partial-mouth periodontal recording survey protocols used in the past. The greatly improved data have allowed us to better identify risk indicators and determinants of periodontitis. For example, we were able to demonstrate that the relationship between periodontitis and diabetes is limited to only severe periodontitis and uncontrolled diabetes, while periodontitis was not associated with obesity.

There is much improvement in the prospects for direct surveys or modeling estimation of periodontitis at both state and local levels, which will open up epidemiologic studies for public health intervention of periodontitis at local levels. Since periodontitis is a public health problem in itself because of its effect on quality of life, but is also associated with other common systemic diseases and conditions, it is of great importance that future prevention and intervention programs and activities can now be based on nationally representative data.Finally, it is possible to monitor the prevalence of periodontitis to assess the progress in reaching the goal of reducing the prevalence of moderate and severe periodontitis in the adult US population proposed by the Healthy People 2020 initiative.

\section{ACKNOWLEDGMENTS}

The authors would like to acknowledge the members of the Centers for Disease Control and Prevention and the American Academy of Periodontology workgroup. ${ }^{12}$ No funding was received for writing this paper.

\section{DISCLAIMER}

The findings and conclusions in this report are those of the authors and do not necessarily represent the official position of the Centers for Disease Control and Prevention.
ORCID

Wenche S. Borgnakke (iD https://orcid.org/0000-0003-3593-093X

\section{REFERENCES}

1. Dye BA, Li X, Thorton-Evans GO. Oral health disparities as determined by selected Healthy People 2020 oral health objectives for the United States, 2009-2010. NCHS Data Brief. 2012; 104:1-8.

2. U. S. Department of Health and Human Services Healthy People 2020. 2010. https://www.healthypeople.gov/2020/topics-objec tives. Accessed April 25, 2019.

3. Brown LJ, Oliver RC, Loe H. Evaluating periodontal status of us employed adults. J Am Dent Assoc. 1990;121(2):226-232.

4. Brown LJ, Brunelle JA, Kingman A. Periodontal status in the United States, 1988-1991: prevalence, extent, and demographic variation. J Dent Res. 1996;75(Spec Iss):672-683.

5. Dye BA, Thornton-Evans GO. A brief history of national surveillance efforts for periodontal disease in the United States. J Periodontol. 2007;78(7 Suppl):1373-1379.

6. Eke PI, Thornton-Evans GO, Wei L, Borgnakke WS, Dye BA. Accuracy of NHANES periodontal examination protocols. J Dent Res. 2010;89(11):1208-1213.

7. Dye BA, Li X, Lewis BG, et al. Overview and quality assurance for the oral health component of the National Health and Nutrition Examination Survey (NHANES), 2009-2010. J Public Health Dent. 2014;74(3):248-256.

8. Eke PI, Dye BA, Wei L, et al. Update on prevalence of periodontitis in adults in the United States: NHANES 2009 to 2012. J Periodontol. 2015;86(5):611-622.

9. Page RC, Eke PI. Case definitions for use in population-based surveillance of periodontitis. J Periodontol. 2007;78(7 Suppl):1387-1399.

10. Eke PI, Page RC, Wei L, Thornton-Evans GO, Genco RJ. Update of the case definitions for population-based surveillance of periodontitis. J Periodontol. 2012;83(12):1449-1454.

11. Eke PI, Dye B. Assessment of self-report measures for predicting population prevalence of periodontitis. J Periodontol. 2009;80(9):1371-1379.

12. Eke PI, Dye BA, Wei L, et al. Prevalence of periodontitis in adults in the United States: 2009 and 2010. J Dent Res. 2012;91(10):914-920.

13. Eke PI, Dye BA, Wei L, et al. Self-reported measures for surveillance of periodontitis. J Dent Res. 2013;92(11):1041-1047.

14. Thornton-Evans GO, Eke PI, Wei L, et al. Periodontitis among adults aged $\geq 30$ years - United States, 2009-2010. MMWR. 2013;62(Suppl 3):129-135.

15. Eke Pl, Zhang $\mathrm{X}, \mathrm{Lu} \mathrm{H}$, et al. Predicting periodontitis at state and local levels in the United States. J Dent Res. 2016;95(5):515-522.

16. Eke PI, Wei L, Borgnakke WS, et al. Periodontitis prevalence in adults $\geq 65$ years of age, in the USA. Periodontol 2000. 2016;72(1):76-95.

17. Eke PI, Thornton-Evans GO, Wei L, et al. Periodontitis in US adults: National Health and Nutrition Examination Survey 2009-2014. J Am Dent Assoc. 2018;149(7):576-588; 588e571-588.e576.

18. Leira Y, Martin-Lancharro P, Blanco J. Periodontal inflamed surface area and periodontal case definition classification. Acta Odontol Scand. 2018;76(3):195-198.

19. Pihlstrom BL, Michalowicz BS, Johnson NW. Periodontal diseases. Lancet. 2005;366(9499):1809-1820.

20. Chapple ILC, Mealey BL, Van Dyke TE, et al. Periodontal health and gingival diseases and conditions on an intact and a reduced periodontium: Consensus report of workgroup 1 of the 2017 World Workshop on the Classification of Periodontal and PeriImplant Diseases and Conditions. J Clin Periodontol. 2018;45(Suppl 20):S68-S77. 
21. Bartold PM, Van Dyke TE. An appraisal of the role of specific bacteria in the initial pathogenesis of periodontitis. J Clin Periodontol. 2019;46(1):6-11.

22. Bartold PM, Van Dyke TE. Host modulation: controlling the inflammation to control the infection. Periodontol 2000. 2017;75(1):317-329.

23. Holtfreter B, Albandar JM, Dietrich T, et al. Standards for reporting chronic periodontitis prevalence and severity in epidemiologic studies: proposed standards from the joint EU/USA periodontal epidemiology working group. J Clin Periodontol. 2015;42(5):407-412.

24. Centers for Disease Control and Prevention (CDC); National Center for Chronic Disease Prevention and Health Promotion; Division of Population Health Behavioral risk factor surveillance system (BRFSS). Atlanta, GA: US Department of Health and Human Services, CDC 2018. http://www.cdc.gov/brfss. Accessed April 25, 2019.

25. Eke PI, Jaramillo F, Thornton-Evans GO, Borgnakke WS. Dental visits among adult Hispanics-BRFSS 1999 and 2006. J Public Health Dent. 2011;71(3):252-256.

26. Blicher B, Joshipura K, Eke P. Validation of self-reported periodontal disease: a systematic review. J Dent Res. 2005;84(10):881-890.

27. Dietrich $\mathrm{T}$, Stosch U, Dietrich $\mathrm{D}$, et al. Prediction of periodontal disease from multiple self-reported items in a German practice-based sample. J Periodontol. 2007;78(7 Suppl):1421-1428.

28. Gilbert GH, Litaker MS. Validity of self-reported periodontal status in the Florida dental care study. J Periodontol. 2007;78(7 Suppl):1429-1438
29. Taylor GW, Borgnakke WS. Self-reported periodontal disease: validation in an epidemiological survey. J Periodontol. 2007;78(7 Suppl):1407-1420.

30. Slade GD. Interim analysis of validity of periodontitis screening questions in the Australian population. J Periodontol. 2007;78(7 Suppl):1463-1470.

31. Cyrino RM, Miranda Cota LO, Pereira Lages EJ, Bastos Lages EM, Costa FO. Evaluation of self-reported measures for prediction of periodontitis in a sample of Brazilians. J Periodontol. 2011;82(12):1693-1704.

32. Eke PI, Lu H, Zhang X, et al. Geospatial distribution of periodontists and US adults with severe periodontitis. J Am Dent Assoc. 2019;150(2):103-110.

33. Centers for Medicare and Medicaid Services. National provider identifier standard (NPI). 2014. https://www.cms.gov/Regulationsand-Guidance/Administrative-Simplification/NationalProvlde ntStand/. Accessed April 25, 2019.

How to cite this article: Eke PI, Borgnakke WS, Genco RJ. Recent epidemiologic trends in periodontitis in the USA. Periodontol 2000. 2020;82:257-267. https://doi.org/10.1111/ prd.12323 